\title{
EVALUATION OF SOIL EROSION AND SUSCEPTIBILITY TO LANDSLIDE MANIFESTATION AS A CONSEQUENCE OF WILDFIRE EVENTS AFFECTED THE ZACHARO MUNICIPALITY, PELOPONNESUS, GREECE
}

\author{
Rozos D. , Lykoudi E. , Tsangaratos P., Markantonis K., Georgiadis P., \\ Rondoyanni Th., Leivaditi A., Kyrousis I.
}

National Technical University of Athens, School of Mining and Metallurgical Engineering, Department of Geological Sciences, 9 Iroon Polytecheiou, 15780,Zografou,Greece,rozos@metal.ntua.gr,mmgpel@central.ntua.gr,ptsag@metal.ntua.gr,markantonis@metal.ntua.gr,pangeor@central.ntua.gr, rondo@central.ntua.gr,alexouli@metal.ntua.gr,kyrousis@metal.ntua.gr

\begin{abstract}
In August 2007, a wildfire affected an area of approximately $135.000 \mathrm{~m}^{2}$ in the Municipality of Zacharo, located in Southern Greece at the Peloponnesus peninsula, causing the loss of 41 human lives, and having a dramatic impact on both ecology and economic-social welfare.

The present study applies a simplified model for the evaluation of soil erosion and the susceptibility to landslide manifestation on the hydrological basins of the Zacharo Municipality, as consequence of wildfire. The methodology was based on the evaluation and analysis of territorial parameters such as lithology, geomorphology, hydrography and land cover, which are thought to be directly or indirectly related to soil erosion.

The final product was a series of hazardous maps showing pre and post-fire soil erosion. An almost double increase in the post-fire area of high vulnerability was identified, covering $48 \%$ of the total area, and affecting dramatically the nearby communities. The outcome of this study helped the local and prefectural authorities to address certain mitigating measures in order to face the disastrous consequences of post-fire soil erosion.
\end{abstract}

Key words: wildfire, soil erosion, landslide manifestation, modelling vulnerability to soil erosion, post-fire management plan, Municipality of Zacharo.

\section{Introduction}

In August 2007, a wildfire affected an area of approximately $135.000 \mathrm{~m}^{2}$ in the Municipality of Zacharo (75\% of the total land area), located in Southern Greece at the Peloponnesus peninsula. It was considered as one of the greatest natural disasters in the history of modern Greece, followed by the loss of 41 human lives and the dramatic impact on both ecology and economic-social welfare. About $70 \%$ of the total cultivated area has been severely burned. According to the Greek office of World Wildlife Fund, the $22.5 \%$ of the Kaiafa Lake and forest, an area of exceptional ecological value, was severely burned. Its unique biodiversity has been harmed to a great degree, while endemic species of both flora and fauna suffered severe damage. 
As a response to that disastrous event a research team from the National Technical University of Athens was formed to help the local Authorities to confront the disastrous effects of wildfire on the environment and the economic and social welfare of the nearby communities, associated with the geological environment and the natural processes that followed the wildfire.

It is well documented that areas affected by wildfire are frequently prone to flooding, landslide manifestation, debris and sediment flows (Gartner et al. 2004), events that are found to be accelerated during the wet season immediately after the wildfire. The parameters that influence the degree in which such events will be triggered are namely: topography, geology, soil characteristics, type of flora, precipitation (amount, intensity and duration) and finally fire severity (Swanson 1981). In general, such extreme events have as a result the removal of plant cover and litter layer, two matters that play an important role in the prevention of soil erosion caused by rainfall impact and overland flow. The consequence of this process is the significant increase in soil vulnerability to erosion processes, while soil alteration in physical and chemical properties is also observed (DeBano et al. 1998). The reactivation of landslide phenomena, mainly destructive debris flows, is also to be expected as a result of high intensity rainfall events, and this will result in blocking drainage pathways, damaging residential structures and network and most importantly endangering human life.

The extend of soil cover and weak rocks that appears along with the recorded high rainfall intensity in the Zacharo area indicate that the manifestation of adverse affects, such as flooding, landslide, debris, and sediment flows, are expected to be very significant and therefore mitigation measures should be implied as soon as possible.

Consequently, the effort of this work was aimed towards identifying, through modelling, the areas of high soil vulnerability to erosion processes and areas prone to landslide manifestation, in order to apply the necessary mitigating measures presenting a valuable post fire erosion control treatment.

\section{Methodology}

The procedure followed during the preparation of a post-fire management plan can be grouped into the following three (3) phases:

a) Collection and process of all available bibliographical data concerning the research area. Mapping and classifying the burned areas according to the burn severity and also verifying and accomplishing a geological map at a scale of 1:25.000, were necessary. Determination and inspection of areas prone to flooding, and areas prone to landsliding.

b) Construction of a valuable database, by processing and digitizing the available data. The data were introduced into a GIS system, in order to construct a variety of thematic maps that would assist the study of the geomorphologic characteristics of the area. Also, a quantitative analysis of the hydrological networks was done and the development of a simplified model for estimating the vulnerability of the formations against soil erosion was established during this phase.

c) Suggestions of mitigating measures in areas of concern and validation of the outcomes of the study will be the aim of this phase.

\section{Geomorphology and Geological settings}

Based on the geotechnical survey carried out (Rozos et al. 2008) the study area morphologically is placed among the basin fold of Zacharo, characterized by a multifold evolution on the account of the 


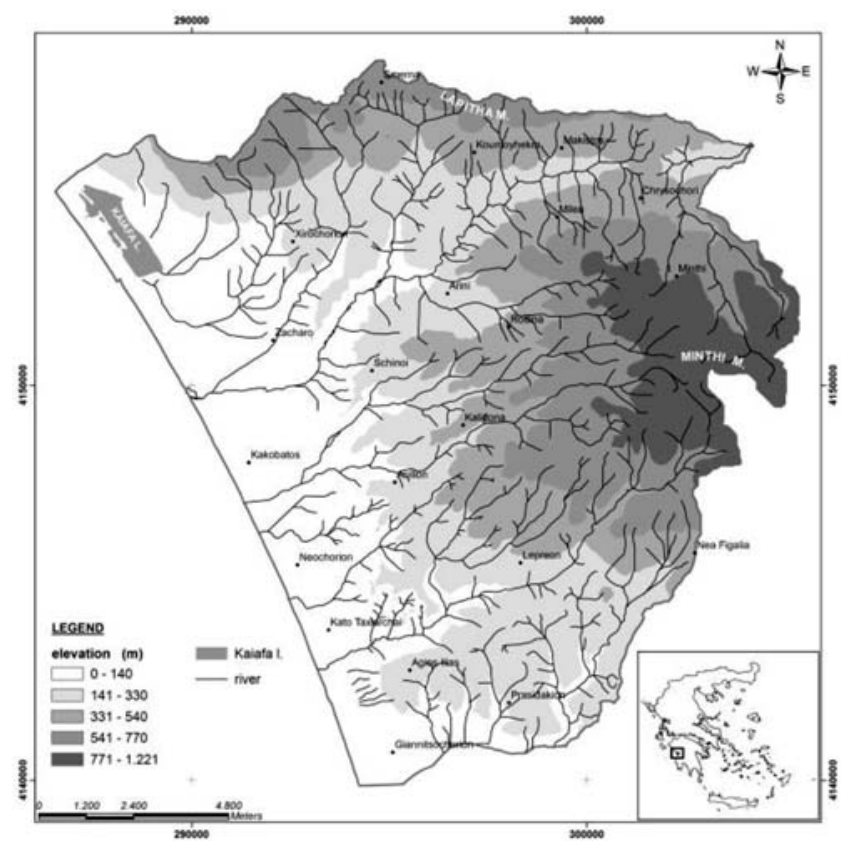

Fig. 1: Morphological Map of the study area.

adjacency with the Ionia trench. Lapithas Mountain to the north and Minthis Mountain to the east are dominant, while a lowland area is observed to the west with small morphological elevation variance, due to the presence of sand-dunes along the coastline. Lake Kaiafa, north to the city of Zacharo, is considered as another dominant morphological element (Fig. 1).

The hydrographic network of the area is relatively dense mainly to the south and west presenting a more complex form, while its type is considered as dentritic $x \alpha \iota$ rectangular, with asymmetry within the hydrological basins. It typically reaches the $4^{\text {th }}$ and $5^{\text {th }}$ class according to Strahler scale while the Kallidonitic River reaches the $6^{\text {th }}$ class. The area has a mean annual precipitation of $900 \mathrm{~mm}$ at the hilly and lowland areas, while in the highlands it is higher than $1000 \mathrm{~mm}$. Also, the mean annual temperature is $17.4^{\circ} \mathrm{C}$, and the mean annual moisture reaches $69 \%$. Finally, the dominant wind direction is southwest.

Geologically the study area consists of alpine and post-alpine formations, which belong to the Ionian, Gavrovon and Pindos Geotectonic zones (IGME 1972; 1974; 1979) (Fig. 2). The formations are limestones Triassic, Jurassic and Cretaceous in age, Schist - Chert formations, transition sediments to flysch and flysch formations. Concerning the post-alpine deposits, Neogene sediments and Quaternary deposits, which appear mainly in the basin of Nedas and Zacharos Rivers, dominate in the hilly and lowland areas. Regarding the permeability of the above formations, they are classified into three categories, namely: a) high permeable, b) low to medium permeable and c) practically impermeable, while a sallow water table is observed within the intense weathering zones.

\section{The Soil Erosion Vulnerability model}

The estimation of the spatial soil vulnerability index and the identification of the prone to landslide manifestation areas was the main objective of the second phase. The spatial soil vulnerability index 


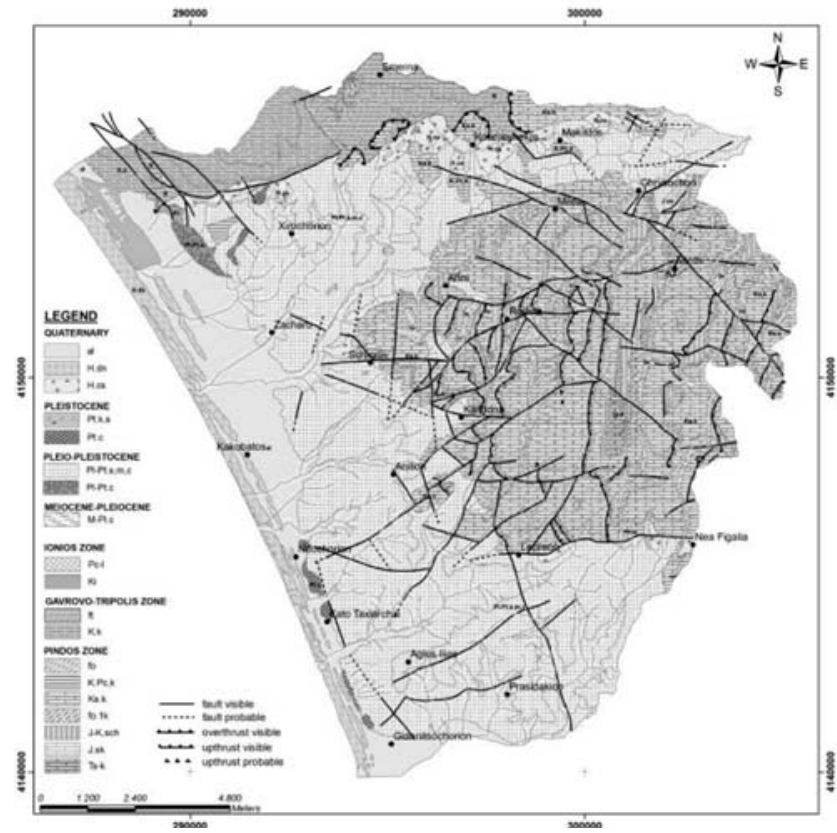

Fig. 2: The geological map of the study area.

was evaluated by applying a simplified model (Alexoudi et al. 2002; Lykoudi et al. 2004) while lithological type, slope gradient, hydrographic texture and vegetation cover were considered as input variables.

A few modifications to the original model were necessary to be done. Specifically, the precipitation parameter was not included in the developed model as it was indirectly calculated by the drainage texture, drainage density and drainage frequency, and also it appeared to have insignificant spatial variation.

However, the most important modification was the input in the original model of a thematic map of "burn severity", which showed the relative amount of damage attributed to an area, as a consequence of wildfire (Key and Benson 2006). It has been observed that in high burn severity areas the probabilities of instability and soil erosion problems are more likely to occur (Benavides-Solorio and MacDonald 2001). The resulting classified map was conducted after field inspection and was coded into three (3) classes of fire severity, namely (a) unburned, (b) moderate burned, and (c) highly burned (Fig. 3).

The classification of the formations into three (3) lithological categories was made according to their likelihood to erode, taking into account the knowledge gained after the field inspection, but also the combined estimation of the lithological texture, the infiltration ability and permeability of the formations in question. In the first category, unweathered intact formations with high permeability, but also those with a weathering mantle of small thickness were classified; In the second category formations which exhibit medium to high infiltration and medium to low permeability value, but also those which are covered by a weathering mantle of medium to big thickness were grouped; In the third category formations that are characterized by high infiltration values and low permeability, and also those which are covered by weathering mantle of great thickness were included. 


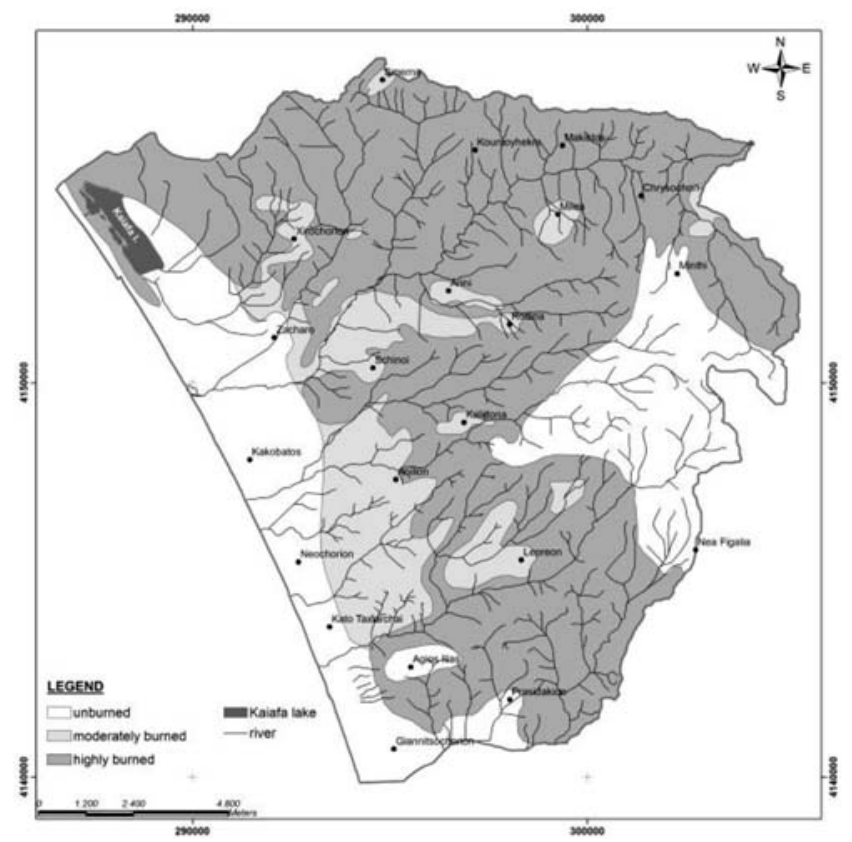

Fig. 3: Burn Severity Map of the Zacharo municipality.

Slope gradient thematic maps were prepared from digital elevation models (DEM) using topographic maps. According to the numerical values of the slope gradient, the research area was classified into three (3) categories: the first one $\left(66.5 \mathrm{~km}^{2}\right)$ with values less than $12 \%$ (gentle slopes), the second $\left(96 \mathrm{~km}^{2}\right)$ with values between 12 and $35 \%$ (medium to high slopes) and finally the third one $\left(43.0 \mathrm{~km}^{2}\right)$ with values greater than $35 \%$ (steep slopes).

The produced map of hydrographic texture is referred to combined evaluation of drainage density and stream frequency parameters that characterize the hydrographic network. Each parameter is classified into three (3) classes, low, medium and high hydrographic density and stream frequency. From the combined evaluation of these two parameters we distinguish two (2) classes: one of low to medium texture, and one of medium to high texture. The first one is characterized by low to medium values of density and frequency while the second one is characterized by medium to high values of density and frequency.

The protection that soil cover provides to an area is considered competitive to the erosion process and in general depends on the type of vegetation. The best protection is provided in areas were densely forest and bushes are identified. On the contrary cultivated areas provide insufficient protection against erosion process, while wastelands are totally unprotected. In the relative map, three (3) classes of soil cover were distinguished: (i) natural rangelands and areas without vegetation cover, (ii) olive groves and other permanent crops and (iii) broad leaved forests and sclerophyllous vegetation.

Based on the above thematic layer maps and the expert knowledge, logical relationship - rules representing boundary conditions of all influential variables on different soil erosion vulnerability values were formulated. Also, by applying spatial analysis techniques a vulnerability map which classifies the research area in low, medium and high vulnerability soil erosion areas was produced (Fig.4). 


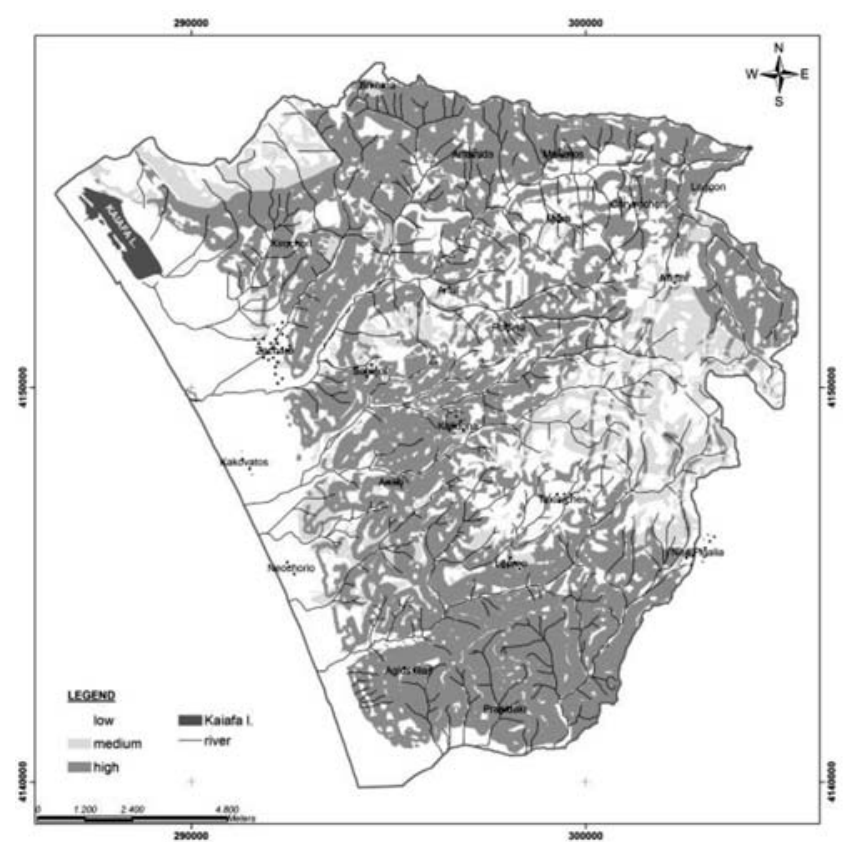

Fig. 4: Vulnerability map of Soil Erosion

\section{Landslide spatial distribution and characteristics}

As already mentioned, areas with high burn severity values would be more likely to exhibit instability problems. Such behaviour could be influenced by several factors that have as a result the reduction of the strength parameters of the formations and the manifestation of landslide or debris flows. The main primary parameters responsible for landslide manifestation throughout the studied area could be grouped as follows:

- The presence of geological formations that are characterized by low geomechanical properties,

- The sequence of geological formations with different geomechanical properties both to the horizontal and vertical extent,

- The intense tectonic action, and seismic activity,

while the main secondary ones are:

- The weathering and erosion process,

- The amount, intensity and duration of precipitation,

- The human intervention, concerning road infrastructure, and cultivation, The last two are the main triggering factors to slope failures manifestation.

The instability problems were mainly connected with the neogene sediments, the flysch formations and the schist-chert series of Pindos zone. They were also observed in highly weathered zones of carbonate formations and in sites where the main discontinuity systems of the rock formations had the same direction to the natural or artificial slopes. They appear in large cultivated areas and on the main road network (Fig.5a \& 5b).

Significant landslides have also been reported to influence populated areas, with main examples the landslides in Kalidona and Lepreo villages. 

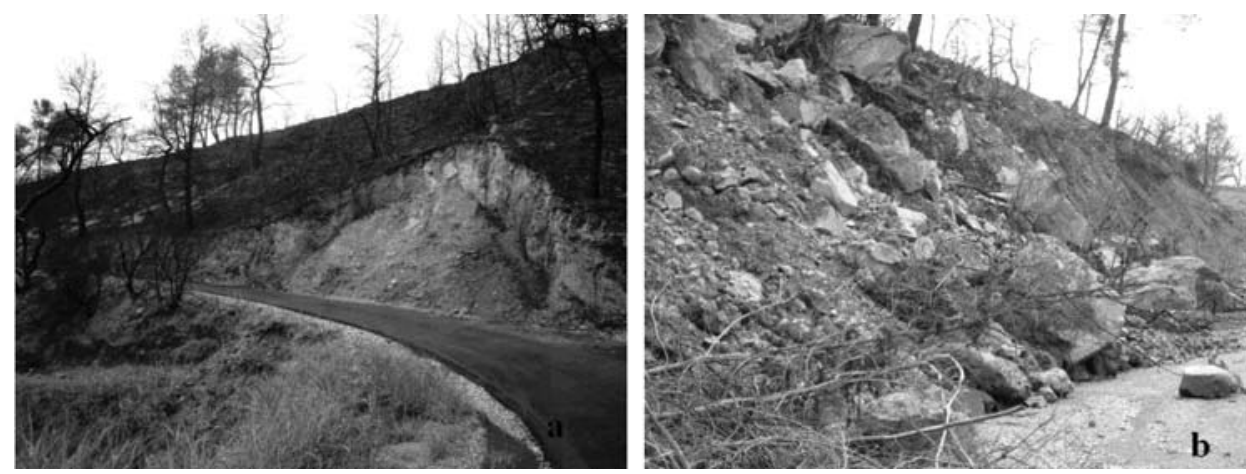

Fig. 5: Significant Landslide phenomena in (a) the road of Koumouthekla - Makistos and (b) outside the Koumouthekla village.

\section{Defining areas of high risk}

By using the developed model, areas characterized by high risk were identified by taking into consideration the susceptibility of soil erosion and landslide manifestation. The area that showed an increase in vulnerability, as a consequence of the August 2007 wildfire, was about $55 \mathrm{~km}^{2}$, i.e. $27 \%$ of the total area, while the $73 \%$ has remained the same. From the area that shows vulnerability increase, about $10.1 \mathrm{~km}^{2}$ (5\% of the total area) changed from low to medium vulnerability, about $16.0 \mathrm{~km}^{2}(8 \%$ of the total area) from low to high vulnerability, and $29.0 \mathrm{~km}^{2}$ (14\% of the total area) from medium to high vulnerability class. The areas that are located mainly at the Northern part of Zacharo municipality (Lapithas mount.), at the central (between Kalidona and Chrisochori village) and the southern part (north of the valley of river Neda) were considered as the most vulnerable ones (Figure 6). Therefore, the suggested mitigation measures should be mainly applied in those areas.

During the study another problem was revealed to the research team. The volumes of water at the exit of the final drain channel downwards the residual area of Zacharo town, compared with the volumes entering the drain channel before the residential area, show a considerable increase. This indicates that the pluvial water of the residential area is added to that of drainage through the sewer channel network. To avoid the flood risk in such cases, the construction of a parallel to the main drain channel or a parametrical one to the town of Zacharo could be the solution.

\section{Mitigation measures - Post fire erosion control treatment}

The implementation of a post-fire erosion control treatment was the main objective of the third phase. The goal of this treatment is to cost-effectively protect the onsite and downstream areas, until the native vegetation germinates, by reducing and delaying the volume of accelerated sediment yield that typically follows a wildfire. The effort was focused in the direction of providing actions that could be followed by the local authorities. The proposed remedial measures were mainly directed towards mitigating the effects of wildfire by taking into consideration the combined evaluation of soil vulnerability index and the zones of landslide instability. Many types of mitigation measures, both on the hillslopes and stream channels were utilized, taking the form of mechanical barriers that retain debris or thick soil covers in place, and reduce the erosive processes caused by rain splash and overland flow, or the form of specific actions that had to be applied before the beginning of the wet season. Those actions included: 


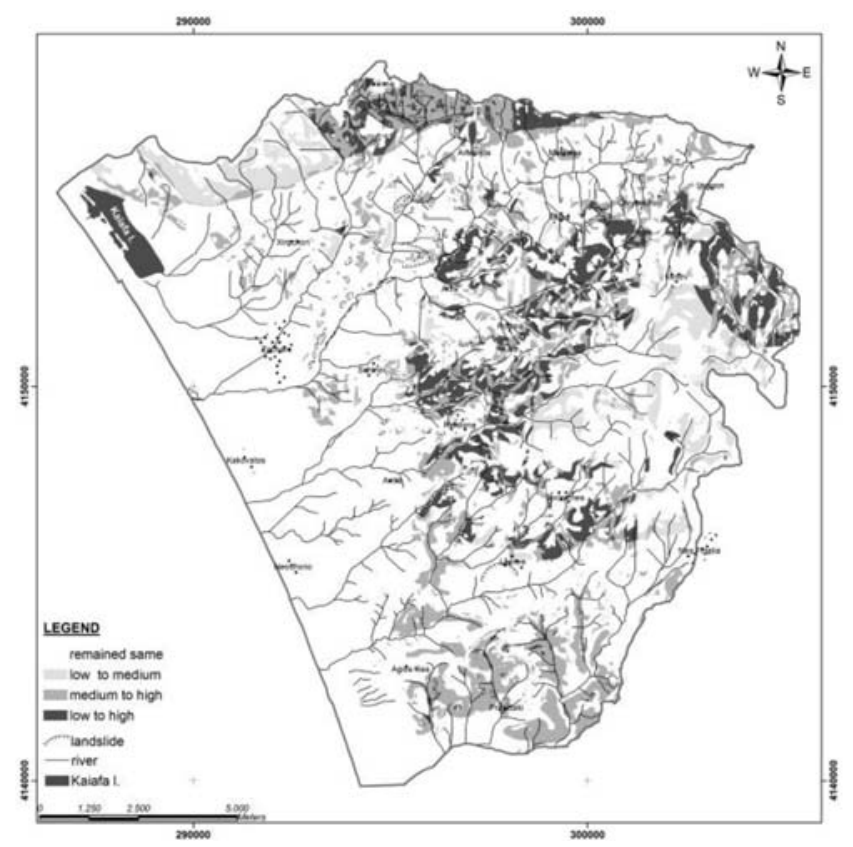

Fig. 6: High risk areas showing the spatial distribution (of what?)

- Immediate installation of an emergency alarm system, that could warn the local authorities in the case of intensive precipitation events, in order to evacuate if necessary the populated areas.

- Construction of log-barriers, and small wooden dams, which retain the material of the eroded soil cover on slopes; thus contributing to the increase of the infiltration rate and reduce of the water runoff velocity.

- Installation of "fences" from steel panels of certain diameter in certain sites along the river path, in order to capture objects that would otherwise block the drainage pathways and act as obstacles forcing the water to flow outside its path.

- Clearance and is some cases widening the main hydrographic network axes.

- Continuous inspection and clearance of the sewer network within the residential area of the Zacharo municipality.

Regarding the construction of log-barriers on slopes, the burned trees should have been cut down, trimmed, and their brunches and/or logs should have been placed on the ground along certain contour lines (Figure 7). As a result, water runoff is not permitted to follow a straight down-slope path. The water is forced to meander back and forth between logs; thus is reducing its velocity and allow water with sufficient time to percolate into the soil. Log-barriers of 3 to $9 \mathrm{~m}$ long and 40 to $60 \mathrm{~cm}$ in diameter should be used in areas of high vulnerability indicated by the model (Fig. 6).

The debris racks (metallic fences) have to be installed in the main drainage pathways and for working effectively, the local authorities are obligated to continuously inspect and when necessary remove the captured material (Fig. 8a \& 8b).

\section{Discussion and Results}

In general, the behavior of geological formations, in response to the natural processes that followed the 


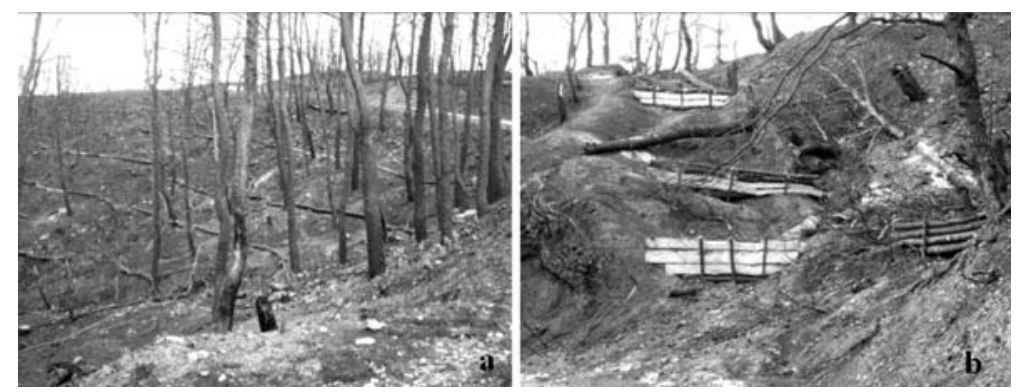

Fig. 7: Log or brunch barriers (a) and small wooden dams (b) for soil erosion protection.
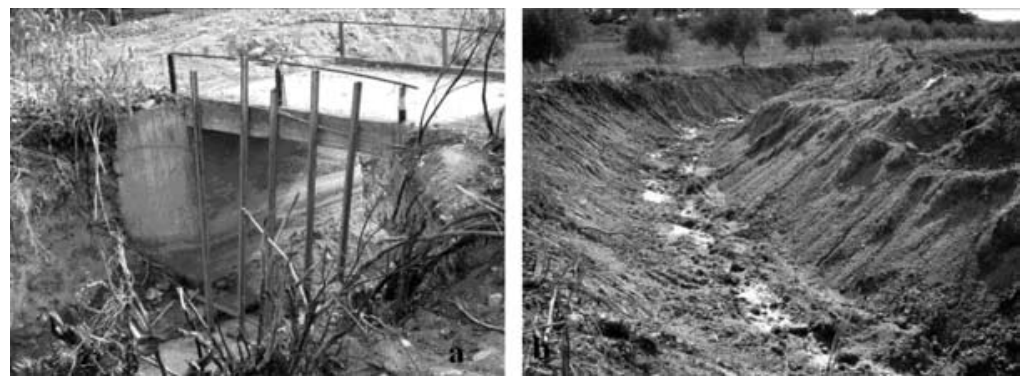

Fig. 8: Debris Rack (metallic fence) (a), widening and clearance of river network (b).

wildfire, depends on their lithological composition, their tectonic history, the susceptibility to the processes of weathering and erosion, along with the geo-mechanical action of ground and surface water.

Furthermore in the examined burned areas, the strength properties of the formations are reduced as a consequence of both, the geo-mechanical action of water (ground and surface) and the changes on the surface layer of the burned area. Specifically the carbonate rocks present a relatively good geomechanical behavior; with rare instability problems (mainly rock falls). The frequent instability problems manifested in flysch, schist-chert and other clastic formations, are creep and various types of slides. In general, landslide manifestation increases in frequency and density in areas where slopes (natural or man-made), have high gradient and in zones of intense weathering. In plio-pleistocene formations altering behaviour could be found, as a consequence of the differences in lithological composition and tectonic distress. In those formations the gradient between the slopes and the dip of the strata is also a parameter that influences their behaviour. Finally the alluvial deposits that cover the lowland area of the municipality of Zacharo (gentle slope gradient) do not exhibit any instability problems.

The implementation of the developed methodology has been helpful in the evaluation of the vulnerability degree of the formations concerning soil erosion. By using GIS system, i) a map of lithological formations prone to erosion, ii) a map of slope gradient (S), iii) a map of hydrographic texture (Y), iv) a map of land use / land cover, and v) a map of burn severity area, were produced. Furthermore, by applying spatial analysis techniques, the vulnerability of formations to soil erosion was calculated in order to produce the final vulnerability map. In particular, severely burned areas with over $35 \%$ of slope gradient, with high hydrographic texture value covered by formations of high infiltration and low permeability values are considered as high vulnerability soil erosion areas. Also 

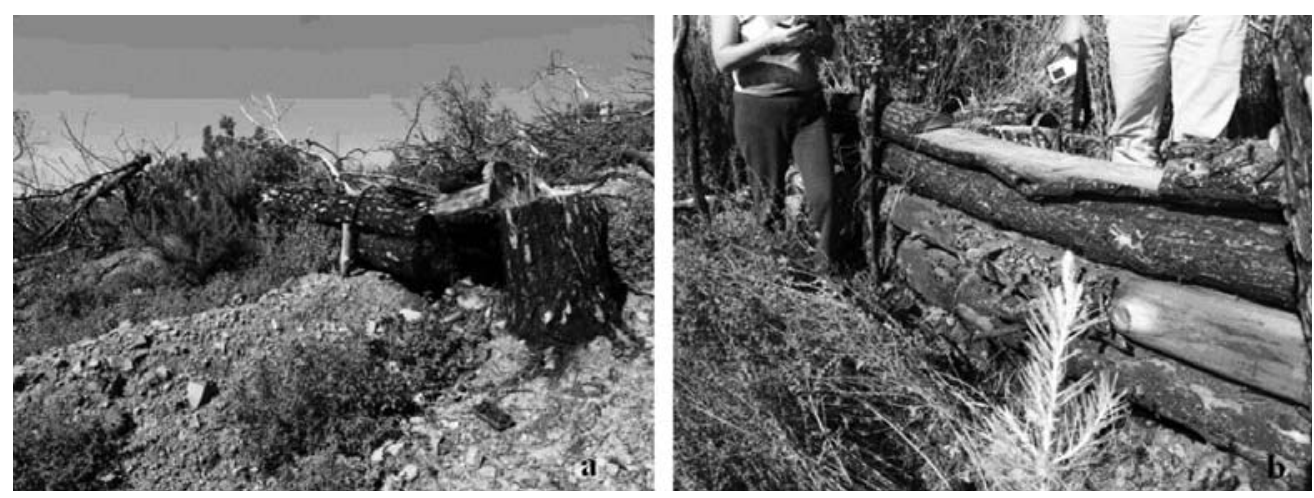

Fig. 9: Failure of log-barrier (a), and effective implementation (b).

those which are covered by weathering mantle of great thickness and are severely burned are considered as high risk areas.

After a wildfire the severity and amount of the disastrous events which affect landscape evolution typically decline in subsequent years as the environment becomes stable. The recovery rate varies and is influenced mainly by the magnitude of the wildfire, the climate, and the soil characteristics of the region in question. These features probably persist longer in arid and semi-arid climates than in humid ones. In the region of Zacharo a medium to high recovery rate was observed. As already mentioned, the area receives a significant amount of precipitation, while the mean annual moisture content reaches $69 \%$, enhancing the vegetation recovery in great parts of the burned area. Parallel to that, the proposed mitigation measures seemed to work well in most of the implemented areas. Their success was also supported by the mild climatic conditions during the first wet season after the wildfire, as well as the relative coordination and efficient actions performed by the local authorities.

Evaluating the applied measures, it was revealed that the grain size of the material produced by soil erosion processes is a significant factor for the effectiveness of the log-barriers. Indeed, large amount of fine-grained material makes the effectiveness of the log-barriers insufficient. The consequences of the present high or low amount of fine-grained soils are shown in Figure 9. Finally, the construction of log-barriers in carbonate formations was found to be inappropriate having in most cases negative influence.

\section{References}

Alexoudi-Livaditi A., Livaditis G., Lykoudi E., 2002. Evaluating the vulnerability to erosion of geological formations and the sediment yield in Lesvos. $6^{\text {th }}$ Hellenic Geographical Conference, Thessaloniki.

Benavides-Solorio, J.; MacDonald, L.H. 2001. Post-fire runoff and erosion from simulated rainfall on small plots, Colorado Front Range. Hydrological Processes. 15:2931-2952.

DeBano, L.F., Neary, D.G., Folliott, P.F., 1998. Fire's Effects on Ecosystems. Wiley, New York.

Gartner, J.E., Bigio, E.R., Cannon, S.H., 2004. Compilation of postwildfire runoff-event data from the western United States. Open- File Report (United States Geological Survey) 04-1085 (http://pubs.usgs.gov/of/2004/1085.html).

IGME publications, Geological map of Greece, scale 1:50.000, (Kyparissia 1979, Kato Figaleia 1974, 
Olympia 1972, and Tropaia 1979 sheets).

Key H., Benson C., 2006. Landscape assessment (LA). In: D.C. Lutes, R.E. Keanne, J.F. Caratti, C.H. Key, N.C. Benson and L.J. Gangi, Editors, FIREMON: Fire Effects Monitoring and Inventory System General Technical Report RMRS-GTR-164-CD. USDA Forestry Service, Fort Collins, Colorado.

Lykoudi E., Zarris D., 2004. The influence of drainage network formation and characteristics over a catchment's sediment yield. $2^{\text {nd }}$ International Conference on Fluvial Hydraulic and Environmental Engineering Girolamo Ippolito, University of Napoli Federico II, Naples, Italy, June 23-25.

Rozos D., Georgiadis P., Alexoudi A., Kyrousis G., Marinos P., Rondogianni T.,Tsiambaos G., Lykoudi E., Markantonis K., Tsangaratos P., 2008. Evaluating soil erosion in the basin of the municipality of Zacharo, Research Team “A” for the fire-stricken areas, NTUA, Athens, Greece.

Swanson J., 1981. Fire and geomorphic processes. in: Mooney, H.A., Bonnicksen, T.M., Christiansen, N.L., Lotan, J.E., Reiners, W.A. (Eds.), Fire Regime and Ecosystem Properties, United States Department of Agriculture, Forest Service, General Technical Report WO vol. 26. United States Government Planning Office, Washington, DC, pp. 401-421. 